\title{
Phosphodiesterase 4 inhibitor plus metformin is superior to metformin alone for the treatment of polycystic ovary syndrome: A rat model study
}

\author{
YUSUF AYTAC TOHMA ${ }^{1}$, GOGSEN ONALAN ${ }^{1}$, MERIH TEPEOGLU ${ }^{2}$, NILUFER BAYRAKTAR ${ }^{3}$, \\ ESER COLAK $^{4}$, EMEL EBRU OZCIMEN $^{4}$ and HULUSI BULENT ZEYNELOGLU ${ }^{1}$
}

Departments of ${ }^{1}$ Obstetrics and Gynecology, ${ }^{2}$ Pathology and ${ }^{3}$ Biochemistry, Başkent University School of Medicine, 06490 Ankara; ${ }^{4}$ Department of Obstetrics and Gynecology, Başkent University School of Medicine, 42080 Konya, Turkey

Received November 26, 2018; Accepted March 19, 2019

DOI: $10.3892 /$ etm.2019.7428

\begin{abstract}
The role of metformin in the management of polycystic ovary syndrome (PCOS) and PCOS-related obesity remains controversial. Recent research on the treatment of PCOS-related obesity investigated novel therapeutic agents with the potential to work synergistically with metformin. The aim of the present study was to determine the synergistic effect of a phosphodiesterase 4 inhibitor (PDE4i) and metformin on weight and hormonal changes in a rat model of PCOS. A total of 40 female Sprague-Dawley rats were randomly divided into 4 groups ( $n=10 /$ group): Sham; PCOS control (no medication after PCOS induction with dehydroepiandrosterone); metformin (300 $\mathrm{mg} / \mathrm{kg} /$ day p.o. after PCOS induction); and metformin + PDE $4 \mathrm{i}(300 \mathrm{mg} / \mathrm{kg} /$ day p.o. metformin $+0.5 \mathrm{mg} / \mathrm{kg} /$ day p.o. PDE4i after PCOS induction). The body weight was measured every 7 days, from day 1 to day 49. Vaginal smears were performed and examined daily via light microscopy for determination of the stage of each rat's estrous cycle. At the end of 21st day and at the end of the study, blood samples were collected from rats and the testosterone and insulin levels were measured. Immunohistochemical staining was performed to quantify phosphorylated cyclic AMP response element-binding protein expression in all groups. At the end of the study, the median body weight differed significantly among the groups $\left(\chi^{2}=30.581, \mathrm{P}<0.001\right)$, being the highest in the PCOS control group and the lowest in the metformin + PDE4i group. At the end of the study, the median testosterone level differed significantly among the groups $\left(\chi^{2}=27.057, \mathrm{P}<0.001\right)$, being the highest in the PCOS
\end{abstract}

Correspondence to: Professor Gogsen Onalan, Department of Obstetrics and Gynecology, Başkent University School of Medicine, Şehit Temel Kuğuoğlu Sk 32 Bahçelievler, Cankaya, Ankara 06490, Turkey

E-mail: gogsenonalan@yahoo.com

Key words: polycystic ovary syndrome, phosphodiesterase 4 inhibitor, metformin, obesity control group and the lowest in the metformin + PDE4i group. The cycle was restored to normal at the end of the study in all the rats in the metformin and metformin + PDE4i groups, whereas an irregular cycle persisted in all the rats in the PCOS control group. In conclusion, PDE4i + metformin was superior to metformin alone in reducing weight gain and decreasing the testosterone levels in a rat model of PCOS.

\section{Introduction}

Polycystic ovary syndrome (PCOS) is a metabolic and hormonal disorder that was first described in 1935 by Stein and Leventhal (1). PCOS is a multifactorial, heterogenous disorder, most commonly affecting women of reproductive age. The worldwide prevalence of this heterogeneous disorder is $2.2-26.7 \%$ (2). PCOS is characterized by hormonal irregularity, including an increased luteinizing hormone (LH) level, a decrease in the follicle-stimulating hormone (FSH) level, hyperandrogenemia and hyperinsulinemia, anovulation and, as a result, menstrual disorders such as oligo/amenorrhea and polycystic ovaries $(3,4)$.

PCOS is a complex syndrome and there are numerous theories regarding its pathophysiology, including impaired androgen, cortisol, lipid and insulin metabolism, as well as chronic subclinical inflammatory processes (5). Chronic ovarian inflammation may cause endocrinological, structural and metabolic changes, ultimately resulting in the development of PCOS $(5,6)$. Animal studies demonstrated that proinflammatory cytokines increase ovarian steroidogenic enzyme levels, leading to an increase in androgen production, whereas anti-inflammatory agents, such as statins and resveratrol, decrease androgen production $(7,8)$. These findings support the hypothesis that PCOS is a proinflammatory disorder, and that chronic subclinical inflammatory processes directly stimulate the development of PCOS-associated metabolic abnormalities and ovulatory dysfunction.

Cyclic nucleotide phosphodiesterases (PDEs) are important for the metabolism of cyclic adenosine monophosphate (cAMP) and cyclic guanosine monophosphate (cGMP), both of which have potent anti-inflammatory properties. The PDE superfamily consists of 11 enzyme subtypes (PDE1-11), some of which hydrolyze cAMP (PDE4, PDE7 and PDE8), whereas 
others hydrolyze cGMP (PDE5, PDE6 and PDE9) (9). PDEs are found in all cells and, in particular, PDE4 and PDE3 are highly expressed in human ovaries and inflammatory cells (10). Inhibition of these enzymes is used to treat chronic inflammatory diseases, such as chronic obstructive pulmonary disease (11). Moreover, the PDE enzymes regulate proinflammatory mediators and the steroid hormone biosynthesis pathway in the ovaries, and may thus play a key role in the pathophysiology of PCOS (12-14).

Metformin has a modest effect in the management of PCOS and PCOS-related obesity (15). Therefore, new therapeutic agents in combination with metformin are needed. PDE-4 inhibitors (PDE4i) which are used for chronic obstructive pulmonary disease have important roles in ovarian steroidogenesis and glucose metabolism (13) and therefore may have a value in the management of PCOS and PCOS-related obesity. However, there is paucity of data in the literature regarding the potential synergistic role of PDE4i in the management of PCOS and PCOS-related obesity $(14,16)$ and there are no data about additive effects of PDE4i on ovarian morphology and phosphorylated cyclic AMP response element binding protein (pCREB) expression (with immunohistochemical staining). Therefore, the aim of the present study was to determine the effect of a PDE4i combined with metformin on ovarian morphology, weight and hormonal changes in a rat model of PCOS.

\section{Materials and methods}

Animals. The present study included 40 female Sprague-Dawley rats (weight, 193-215 g) supplied by the Başkent University Experimental Animal Center (Ankara, Turkey). All the rats were housed in standard cages at $23^{\circ} \mathrm{C}, 50-60 \%$ humidity and a 12-h light and dark cycle, with free access to food and water. The study protocol was approved by the Başkent University Animal Care and Use Committee (project no. DA15/45), in accordance with Turkish legislation for animal experimentation.

Study groups. The rats were randomly divided into 4 groups ( $\mathrm{n}=10$ /group) as follows: Sham, PCOS control, metformin and metformin + PDE4i.

Sham group. Rats in the sham group were injected with $0.2 \mathrm{ml}$ sesame oil for 21 days (days 1-21), after which time they received only distilled water via orogastric gavage for the next 28 days.

PCOS control group. Rats in the PCOS control group received daily subcutaneous (SC) injections of dehydroepiandrosterone (DHEA; Biosteron $25 \mathrm{mg}$; Lekam Pharmaceutical, Zakroczym, Poland) at $60 \mathrm{mg} / \mathrm{kg} / \mathrm{day}$ dissolved in $0.1 \mathrm{~m}$ sesame oil for 21 days (days 1-21) to induce PCOS as described by Anderson et al (17). Hyperandrogenemia and irregular cycle/anovulation based on vaginal smear examination were considered as successful PCOS induction $(18,19)$. After induction of PCOS, the rats were given only distilled water via orogastric gavage for the next 28 days; they did not receive any drugs.

Metformin group. After induction of PCOS, the rats were administered metformin (Glucophage $500 \mathrm{mg}$; Merck
Pharmaceuticals, Semoy, France) at $300 \mathrm{mg} / \mathrm{kg} / \mathrm{day}$ via orogastric gavage for the next 28 days. The metformin dose used in this study was equivalent to that used in the treatment of PCOS patients $(20,21)$.

Metformin + PDE4i group. After induction of PCOS, the rats were administered metformin (Glucophage $500 \mathrm{mg}$; Merck Pharmaceuticals) at $300 \mathrm{mg} / \mathrm{kg} /$ day plus PDE4i (Daxas 500 mcg; Takeda Pharmaceuticals, Osaka, Japan) at $0.5 \mathrm{mg} / \mathrm{kg}$ day via orogastric gavage for the next 28 days. The PDE4i dose used in this study was equivalent to that used in the treatment of PCOS patients $(14,21)$.

\section{Sample collection and data recording}

Body weight. Body weight was measured every 7 days, from day 1 to day 49 .

Vaginal smears. Vaginal smears were performed and examined daily under a light microscope for determination of the stage of each rat's estrous cycle, based on the dominant cell types as follows: Pro-estrus smear, round and nucleated epithelial cell types; estrus smear, cornified squamous epithelial cells; metestrus smear, cornified squamous epithelial cells and a predominance of leukocytes; and diestrus smear, nucleated epithelial cells and a predominance of leukocytes. The ovulation of female rats occurs from the onset of pro-estrus phase to the end of estrus phase, and the mean cycle length of female rats is 4 days. The phase sequence pro-estrus, estrus, metestrus and diestrus was considered as regular. Irregular cycles were characterized by remaining in the same phase for 4-5 days, or if the phases did not follow the sequence mentioned above. Due to these properties, rats are considered as an optimal model for investigating changes that occur throughout the reproductive cycle (22) (Fig. 1).

Serum and ovaries. At the end of 21st day, blood samples were collected from the heart of all rats and centrifuged for $15 \mathrm{~min}$ at 2,500 rpm. The upper layer of serum was transferred into an Eppendorf tube and stored at $-80^{\circ} \mathrm{C}$. At the end of the study, all rats were anesthetized with $40 \mathrm{mg} / \mathrm{kg}$ ketamine hydrochloride (Ketosal; Interhas Co. Ltd., Ankara, Turkey) and $5 \mathrm{mg} / \mathrm{kg}$ of xylazine hydrochloride (Xylazin Bio, Interhas Co. Ltd.) via intraperitoneal injection, and euthanized by cervical dislocation after heart blood sampling via cardiac puncture. Samples of blood were taken from the ventricle of heart by accessed either via the left side of the chest, through the diaphragm, from the top of the sternum. Death of rats were confirmed by heart beat (assessed for five minutes or more), pupillary response to light (dilated and unresponsive to light) and respiratory pattern (lack of spontaneous breathing). In all rats, 5-7 $\mathrm{ml}$ of blood was available for collection and was centrifuged for $15 \mathrm{~min}$ at 2,500 $\mathrm{rpm}$. The upper serum layer was transferred into an Eppendorf tube and stored at $-80^{\circ} \mathrm{C}$. Next, 1 ovary from each rat was removed and fixed in $4 \%$ paraformaldehyde, whereas the other ovary was removed, frozen in liquid nitrogen, and then stored at $-80^{\circ} \mathrm{C}$ for further analysis.

Testosterone and insulin measurement. The serum testosterone $(\mathrm{ng} / \mathrm{ml})$ and insulin levels (uIU/ml) were determined by ELISA kits (Cusabio Biotech. Co., Ltd., Wuhan, China) 

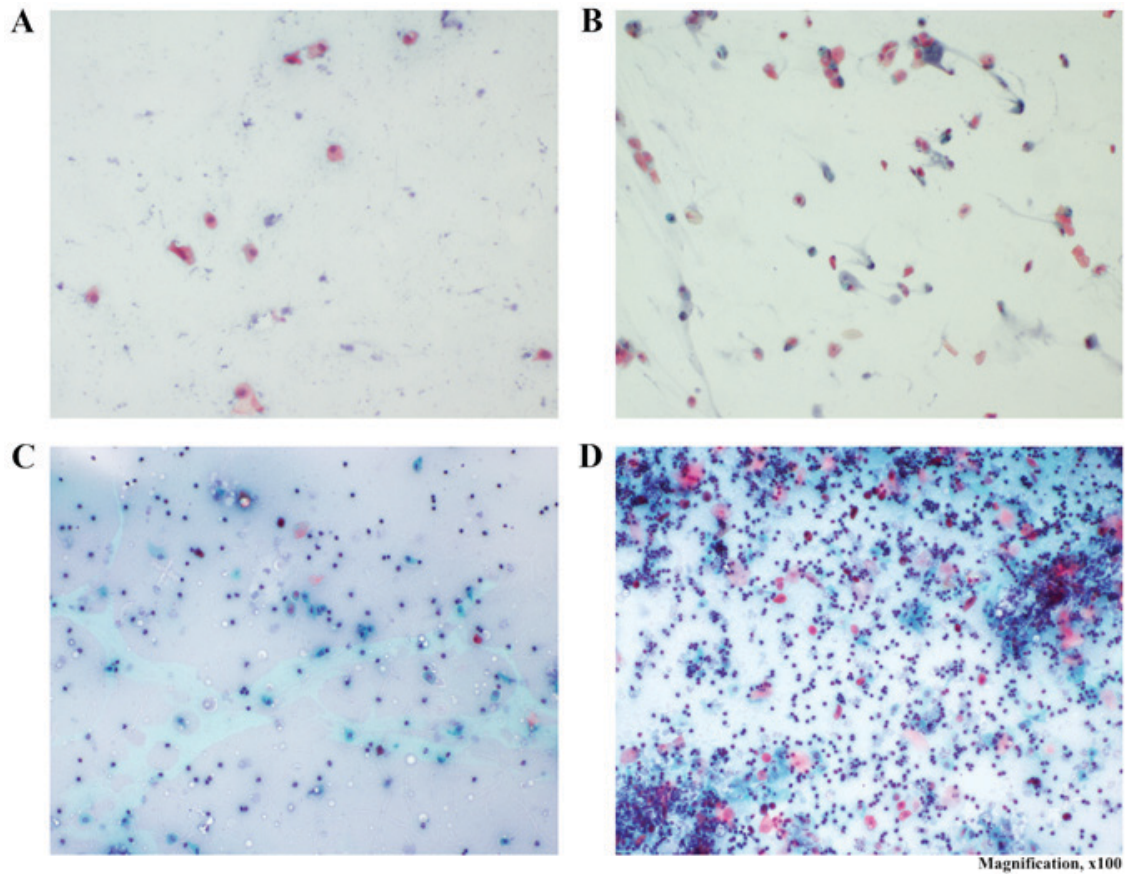

Figure 1. Vaginal smears of rats. (A-D) Pro-estrus, estrus, metestrus and diestrus in the control group (Papanicolaou stain; magnification, x100). (A) Pro-estrus: Round and nucleated epithelial cell types were mainly observed. (B) Estrus: Cornified squamous epithelial cells were mainly observed. (C) Metestrus: Cornified squamous epithelial cells were mainly observed; there was also leukocyte predominance. (D) Diestrus: Nucleated epithelial cells were mainly observed; there was also leukocyte predominance.

and only the testosterone and insulin levels were measured, as some studies have reported that DHEA-induced PCOS in rats is associated with excess androgen, $\mathrm{LH}$ hypersecretion, an irregular estrous cycle, and an elevated insulin level, whereas others reported no change in LH and FSH levels $(8,23-25)$.

Ovarian morphology and immunohistochemistry. The ovaries from the rats in the all groups were removed, processed and embedded in paraffin blocks. Hematoxylin and eosin staining was performed and slides were assayed. Immunohistochemical staining was performed to quantify phosphorylated cyclic AMP response element-binding protein (pCREB) expression in all groups. The intensity of pCREB expression was graded as follows: Negative, 0; weak, 1 ; moderate, 2 ; and strong, 3 . The percentage of positively stained cells was also recorded. The staining pattern was evaluated as follows: High expression, $\geq 50 \%$ of cells exhibiting moderate-strong intensity; and low expression, $<50 \%$ of cells exhibiting moderate-strong intensity (26).

Statistical analysis. Data were analyzed using MS-Excel 2010 for Windows and IBM SPSS Statistics for Windows v.22.0 (IBM Corp., Armonk, NY, USA). The normality of the distribution of continuous variables (weight, testosterone and insulin) was graphically and statistically evaluated using the Shapiro-Wilk test; none of the variables were normally distributed. Median [IQR (interquartile range)] values were used to represent descriptive statistics. Kruskal-Wallis non-parametric variance analysis $\left(\chi^{2}\right)$ was used to compare median body weight, testosterone and insulin values among groups. In order to find different groups, Bonferroni corrected Mann-Whitney test was used in post-hoc pairwise comparisons. The Wilcoxon's signed rank test was used to compare values at baseline (day 1) and at the end of the study. The level of statistical significance was set at $\mathrm{P}<0.05$.

\section{Results}

Animals. The study was initiated with 40 rats randomly distributed into 4 groups ( $n=10 /$ group); however, 1 rat in the sham group, 1 rat in the PCOS control group, and 1 rat in the metformin group died before day 21, and 1 rat in the metformin group was excluded from the study due to failure to induce PCOS. The study was completed with 36 rats: $9(25.0 \%)$ in the sham group, $9(25 \%)$ in the PCOS control group, $8(22.2 \%)$ in the metformin group, and $10(27.8 \%)$ in the metformin + PDE4i group.

Body weight at baseline. At baseline (day 1) there was no significant difference in the median body weight among the groups $\left(\chi^{2}=0.615, P=0.893\right)$. Body weight was equally distributed in the study groups (Table I).

Estrous cycle and induction of PCOS. PCOS induction was determined based on assessment of 8-day smear results from day 14 to day 21 and androgen levels; hyperandrogenemia and irregular cycle were considered as successful PCOS induction $(18,19)$. All 9 rats in the sham group had a normal cycle, whereas irregular cycles were observed in all the rats in the PCOS control $(n=9)$ and metformin + PDE4i $(n=10)$ groups. An irregular estrous cycle was observed in 8 of the rats in the metformin group, whereas 1 rat had a regular cycle and was excluded from the study due to failure to induce PCOS. The study continued as designed after confirmation of PCOS induction (Fig. 2). 
Body weight after induction of PCOS (day 21). The median body weight on day 21 differed significantly among groups $\left(\chi^{2}=18.672, \mathrm{P}<0.001\right)$. Based on post hoc binary comparison performed to identify between-group differences, all treatment groups had a higher median body weight compared with the sham group on day $21(\mathrm{P}<0.01)$, whereas the median body weight did not differ significantly between the 3 study groups ( $\mathrm{P}>0.05)$ (Table I and Fig. 3).

Testosterone and insulin levels following induction of PCOS. Testosterone and insulin levels were measured in all groups on day 21 . The median testosterone on day 21 differed significantly among groups $\left(\chi^{2}=19.533, \mathrm{P}<0.001\right)$. Post hoc test results revealed that the median testosterone level in the 3 study groups was significantly higher compared with that in the sham group on day $21(\mathrm{P}<0.05)$, whereas there was no significant difference among the 3 study groups $(\mathrm{P}>0.05)$ (Table II; Fig. 4A). The median insulin level also differed significantly among groups $\left(\chi^{2}=21.864, \mathrm{P}<0.001\right)$. The median insulin level was significantly lower in the sham group compared with the 3 study groups on day $21(\mathrm{P}<0.05)$, but did not differ significantly among the 3 study groups ( $>>0.05$ ) (Table III; Fig. 4B).

Body weight at the end of the study. At the end of the study, median body weight differed significantly among groups $\left(\chi^{2}=30.581, \mathrm{P}<0.001\right)$. Post hoc binary comparison performed to identify between-group differences revealed that there was a significant difference between the metformin + PDE4i and metformin alone groups $(Z=3.284, P=0.006)$, between the metformin + PDE4i and PCOS control groups $(Z=5.147$, $\mathrm{P}<0.001)$, and between the sham and PCOS control groups $(\mathrm{Z}=3.783, \mathrm{P}=0.001)$. At the end of the study, median body weight was the highest in the PCOS control group, followed by the metformin, sham and metformin + PDE4i groups (Table I; Fig. 3).

Testosterone level at the end of the study. At the end of the study, the median testosterone level differed significantly among groups $\left(\chi^{2}=27.057, \mathrm{P}<0.001\right)$. Post hoc binary comparison performed to identify between-group differences revealed that there was a significant difference between the metformin + PDE4i and metformin alone groups $(\mathrm{Z}=2.697$, $\mathrm{P}=0.042$ ), between the metformin + PDE4i and PCOS control groups $(\mathrm{Z}=4.876, \mathrm{P}<0.001)$, and between the sham and PCOS control groups $(\mathrm{Z}=3.782, \mathrm{P}=0.001)$. At the end of the study, the testosterone level was the highest in the PCOS control group, followed by the metformin, sham, and metformin + PDE4i groups (Table II; Fig. 4A). As compared to day 21, the testosterone level had changed significantly in the 3 study groups at the end of the study $(\mathrm{P}<0.05)$, but not in the sham group $(\mathrm{Z}=0.415, \mathrm{P}=0.678)$. The testosterone level was increased in 8 of the 9 rats in the PCOS control group at the end of the study, as compared to day 21 , whereas the testosterone level was lower at the end of the study compared with that on day 21 in all the rats in the metformin and metformin + PDE4i groups.

Insulin level at the end of the study. At the end of the study, the median insulin level differed significantly among groups $\left(\chi^{2}=25.347, \mathrm{P}<0.001\right)$. Based on post hoc binary comparison performed to identify between-group differences, there were

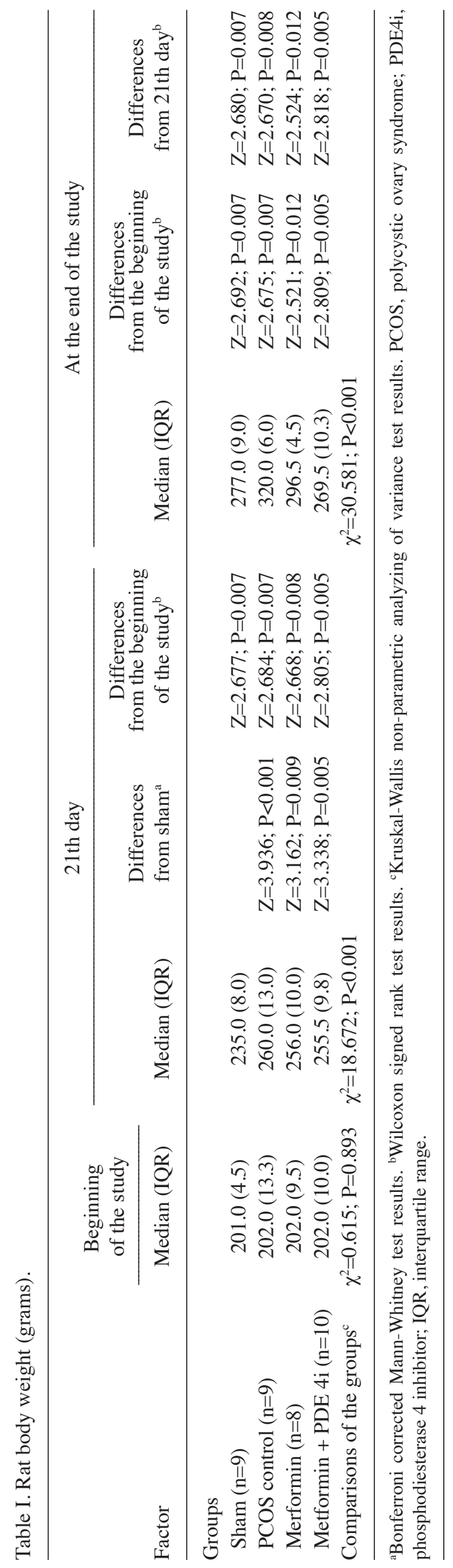


A
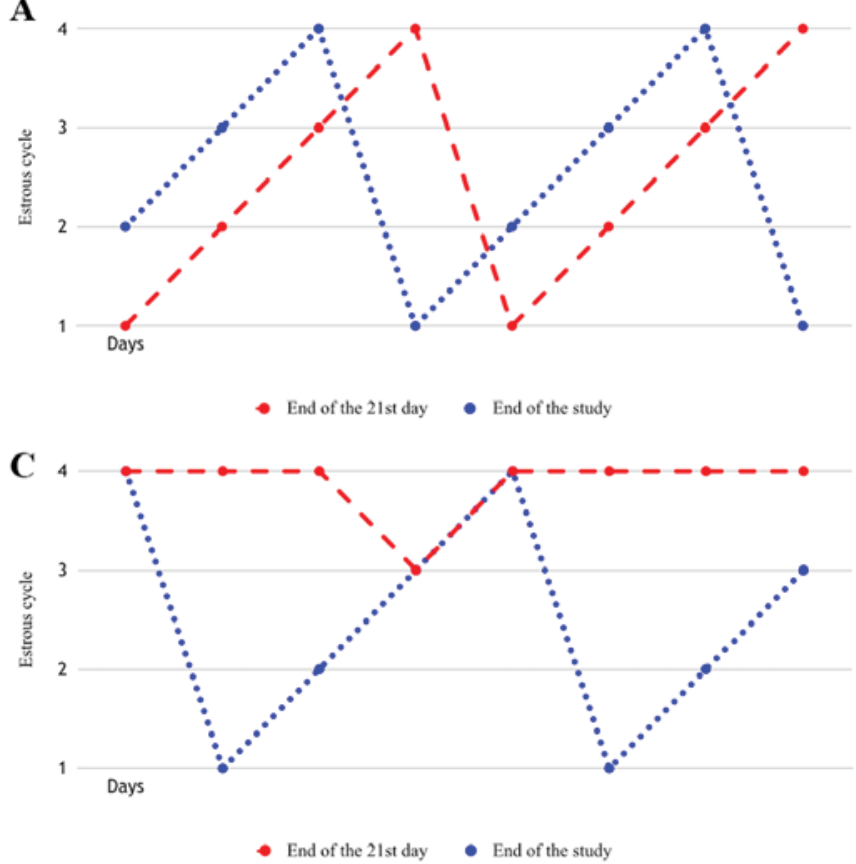

B

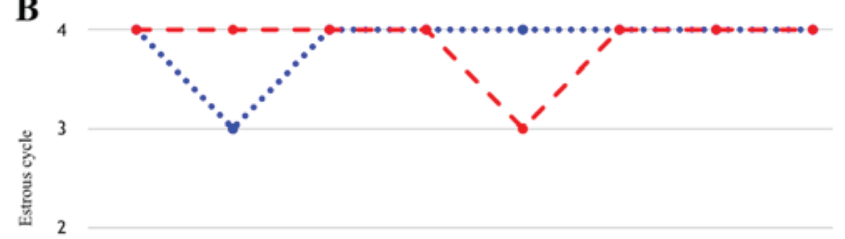

Days

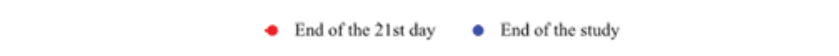

D

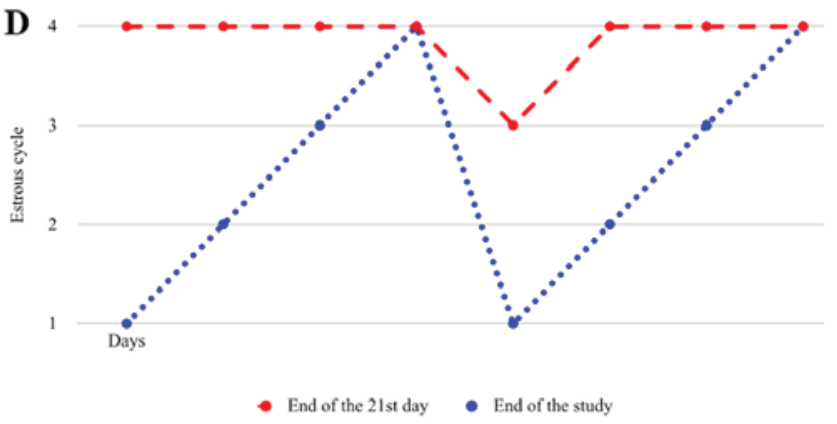

Figure 2. Estrous cycle pattern of a randomly selected rat from each group at end of the 21st day and at the end of the study. (A) Sham group. (B) PCOS control group. (C) Metformin group. (D) Metformin + PDE4i group. Estrous cycles (y axis): 1, pro-estrus; 2, estrus; 3, metestrus; 4, diestrus. PCOS, polycystic ovary syndrome; PDE4i, phosphodiesterase 4 inhibitor.

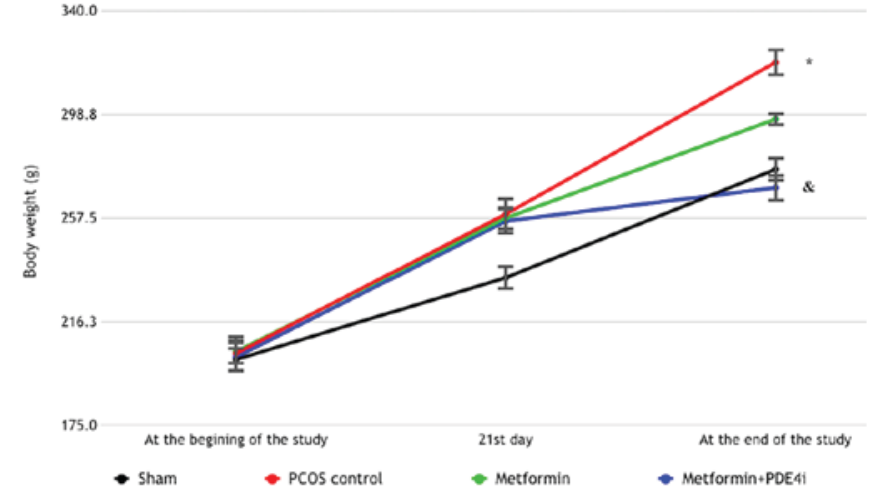

Figure 3. Changes in body weight of groups during the study period. $* \mathrm{P}<0.001$ compared with the Metformin + PDE4i group (at the end of the study); ${ }^{\&} \mathrm{P}=0.006$ compared with the Metformin group (at the end of the study). PCOS, polycystic ovary syndrome; PDE4i, phosphodiesterase 4 inhibitor.

significant differences between the metformin + PDE4i and PCOS control groups $(\mathrm{Z}=4.958, \mathrm{P}<0.001)$, and between the metformin and PCOS control groups $(\mathrm{Z}=3.199, \mathrm{P}=0.008)$. At the end of the study, the median insulin level was the highest in the PCOS control group, followed by the sham, metformin, and metformin + PDE4i groups. As compared to day 21, at the end of the study the change in the median insulin level was not significant in the PCOS control group $(\mathrm{Z}=0.059, \mathrm{P}=0.953)$, whereas the change was significant in all other groups $(\mathrm{P}<0.05)$ (Table III; Fig. 4B). The insulin level was increased in 7 of the 9 rats in the sham group at the end of the study, as compared to day 21, whereas the insulin level was lower at the end of the study in all rats in the metformin and metformin + PDE4i groups compared with day 21 .
Estrous cycle at the end of the study. Based on vaginal smear results in all rats on the last 8 days of the study, all 9 rats in the sham group had a normal cycle at the end of the study. The cycle in all the rats in the metformin $(n=8)$ and metformin + PDE4i $(n=10)$ groups was restored to normal at the end of the study, whereas an irregular cycle persisted in all the rats in the PCOS control group $(n=9)$.

Ovarian morphology and immunohistochemistry at the end of the study. Normal ovarian morphology was observed in the ovarian sections of the sham, metformin and metformin + PDE4 groups, whereas numerous cystic follicles were identified in the ovarian sections of the PCOS control group (Fig. 5).

Low expression staining pattern was observed in all the samples obtained from the rats in the sham and PCOS control groups, whereas high expression staining pattern was observed in $1(12.5 \%)$ rat in the metformin group and four $(40.0 \%)$ rats in the merformin + PDE4i group (Fig. 6).

\section{Discussion}

The present study yielded two important findings. First, the combination of PDE4i and metformin was superior to metformin alone in reducing weight gain in rats with PCOS, which is likely associated with the effects of PDE4i on lipid, insulin and glucose metabolism. Second, the observed decrease in the testosterone level tended to be greater in rats with PCOS that received a combination of PDE4i and metformin, as compared to metformin alone. To the best of our knowledge, this is the first study using a rat model of PCOS to investigate the effects of PDE4i on weight gain, endocrine parameters, such as testosterone and fasting insulin level, and the estrous cycle. In addition, our results including 
Table II. Testosterone levels.

\begin{tabular}{|c|c|c|c|c|}
\hline \multirow[b]{2}{*}{ Factor } & \multicolumn{2}{|c|}{ 21th day } & \multicolumn{2}{|c|}{ At the end of the study } \\
\hline & Median (IQR) & $\begin{array}{l}\text { Differences } \\
\text { from sham }{ }^{\mathrm{a}}\end{array}$ & Median (IQR) & $\begin{array}{l}\text { Differences } \\
\text { from 21th day }\end{array}$ \\
\hline \multicolumn{5}{|l|}{ Groups } \\
\hline Sham $(n=9)$ & $1.07(1.04)$ & & $1.22(0.66)$ & $\mathrm{Z}=0.415 ; \mathrm{P}=0.678$ \\
\hline PCOS control $(n=9)$ & $4.40(0.61)$ & $\mathrm{Z}=3.474 ; \mathrm{P}=0.003$ & $4.88(0.65)$ & $\mathrm{Z}=2.547 ; \mathrm{P}=0.011$ \\
\hline Merformin $(n=8)$ & $4.35(0.95)$ & $\mathrm{Z}=2.821 ; \mathrm{P}=0.015$ & $1.67(0.36)$ & $\mathrm{Z}=2.521 ; \mathrm{P}=0.012$ \\
\hline Metformin + PDE 4 inhibitor $(n=10)$ & $4.69(1.85)$ & $\mathrm{Z}=4.109 ; \mathrm{P}<0.001$ & $1.05(0.22)$ & $\mathrm{Z}=2.803 ; \mathrm{P}=0.005$ \\
\hline Comparisons of the groups ${ }^{c}$ & $\chi^{2}=19.533 ; \mathrm{P}<0.001$ & & $\chi^{2}=27.057 ; \mathrm{P}<0.001$ & \\
\hline
\end{tabular}

${ }^{a}$ Bonferroni corrected Mann-Whitney test results. ${ }^{b}$ Wilcoxon signed rank test results. ${ }^{c}$ Kruskal-Wallis non-parametric analyzing of variance test results. PCOS, polycystic ovary syndrome; PDE4i, phosphodiesterase 4 inhibitor; IQR, interquartile range.

Table III. Insulin levels.

\begin{tabular}{|c|c|c|c|c|}
\hline \multirow[b]{2}{*}{ Study groups } & \multicolumn{2}{|c|}{21 th day } & \multicolumn{2}{|c|}{ At the end of the study } \\
\hline & Median (IQR) & $\begin{array}{l}\text { Differences } \\
\text { from sham }^{\mathrm{a}}\end{array}$ & Median (IQR) & $\begin{array}{c}\text { Differences } \\
\text { from 21th day }\end{array}$ \\
\hline \multicolumn{5}{|l|}{ Groups } \\
\hline Sham $(n=9)$ & $117.32(11.29)$ & & $119.92(14.15)$ & $\mathrm{Z}=2.310 ; \mathrm{P}=0.021$ \\
\hline PCOS control $(n=9)$ & $198.78(15.17)$ & $\mathrm{Z}=4.573 ; \mathrm{P}<0.001$ & $194.52(18.91)$ & $\mathrm{Z}=0.059 ; \mathrm{P}=0.953$ \\
\hline Merformin $(n=8)$ & $184.57(28.14)$ & $\mathrm{Z}=2.918 ; \mathrm{P}=0.012$ & $106.63(53.97)$ & $\mathrm{Z}=2.521 ; \mathrm{P}=0.012$ \\
\hline Metformin + PDE 4 inhibitor $(n=10)$ & $177.83(28.69)$ & $\mathrm{Z}=3.085 ; \mathrm{P}=0.006$ & $97.12(17.52)$ & $\mathrm{Z}=2.803 ; \mathrm{P}=0.005$ \\
\hline Comparisons of the groups ${ }^{c}$ & $\chi^{2}=21.864 ; \mathrm{P}<0.001$ & & $\chi^{2}=25.347 ; \mathrm{P}<0.001$ & \\
\hline
\end{tabular}

${ }^{a}$ Bonferroni corrected Mann-Whitney test results. ${ }^{b}$ Wilcoxon signed rank test results. ${ }^{c}$ Kruskal-Wallis non-parametric analyzing of variance test results. PCOS, polycystic ovary syndrome; PDE4i, phosphodiesterase 4 inhibitor; IQR, interquartile range.

the significant synergistic effects of a PDE4i to metformin on weight loss and endocrine parameters such as testosterone, will encourage design human studies about using PDE4i in obese PCOS patients for obtain sufficient evidence to the use of clinical practice.

Stimulation of $\beta$-adrenergic receptors and the adenylate cyclase complex in fat cell membranes can cause production of cAMP, which causes activation of a cAMP-dependent protein kinase that induces lipolysis (27). Agents that increase the cAMP level and are used for weight control include forskolin and PDE inhibitors (28). It was reported that PDE4i, which specifically increases cAMP instead of cGMP, can cause lipolysis and plays an important role in fat metabolism $(23,29)$. In a rat model study by Nakamura et al $(30)$, a PDE4i decreased cytoplasmic PDEs and increased lipolysis in adipose tissue in rats that were fed, but the same effect was not observed in rats that were not fed, indicating that PDE4i exerts a lipolytic effect that increases with food intake and obesity. Zhang et al (31) investigated the role of PDE4B enzymes (the major PDE4 isoform) in regulating the inflammatory response in the inflammation of adipocytes and energy balance. They reported that PDE4B-null mice had less adipose tissue with an elevated cAMP level and lower serum leptin levels, indicating that PDE4B inhibitors may be useful for the treatment of obesity and suppression of obesity-induced inflammation in white adipose tissue. PDE4i also reduces body weight in humans $(14,32)$. Jensterle et al (16) reported that PDE4i + metformin was superior to metformin alone for reducing body weight in humans $(4.2-0.9 \mathrm{~kg}$ ), noting that the primary cause of the observed effect was associated with lipolysis and reduced fat mass. Another study by Ong et al (32) demonstrated that liraglutide [glucagon-like peptide-1 (GLP-1) analogue] was superior to PDE4i for reducing body weight in humans, but PDE4i was more efficacious compared with metformin.

PDE4 enzymes play an important role in glucose metabolism (33), and Vollert et al used a rat model of type 2 diabetes to examine the effects of PDE4i on GLP-1 and insulin, both of which are important hormones for glucose metabolism. Their results demonstrated that PDE4i protects against diabetes via two important mechanisms: i) Increased secretion of intestinal GLP-1, which decreases the serum glucose level via increasing the secretion of insulin; and ii) enhanced secretion of insulin in the pancreas as a result of its protective effect on pancreatic islets (33). As such, the effect of PDE4i on glucose metabolism may be due to the increase in the GLP-1 level, which 

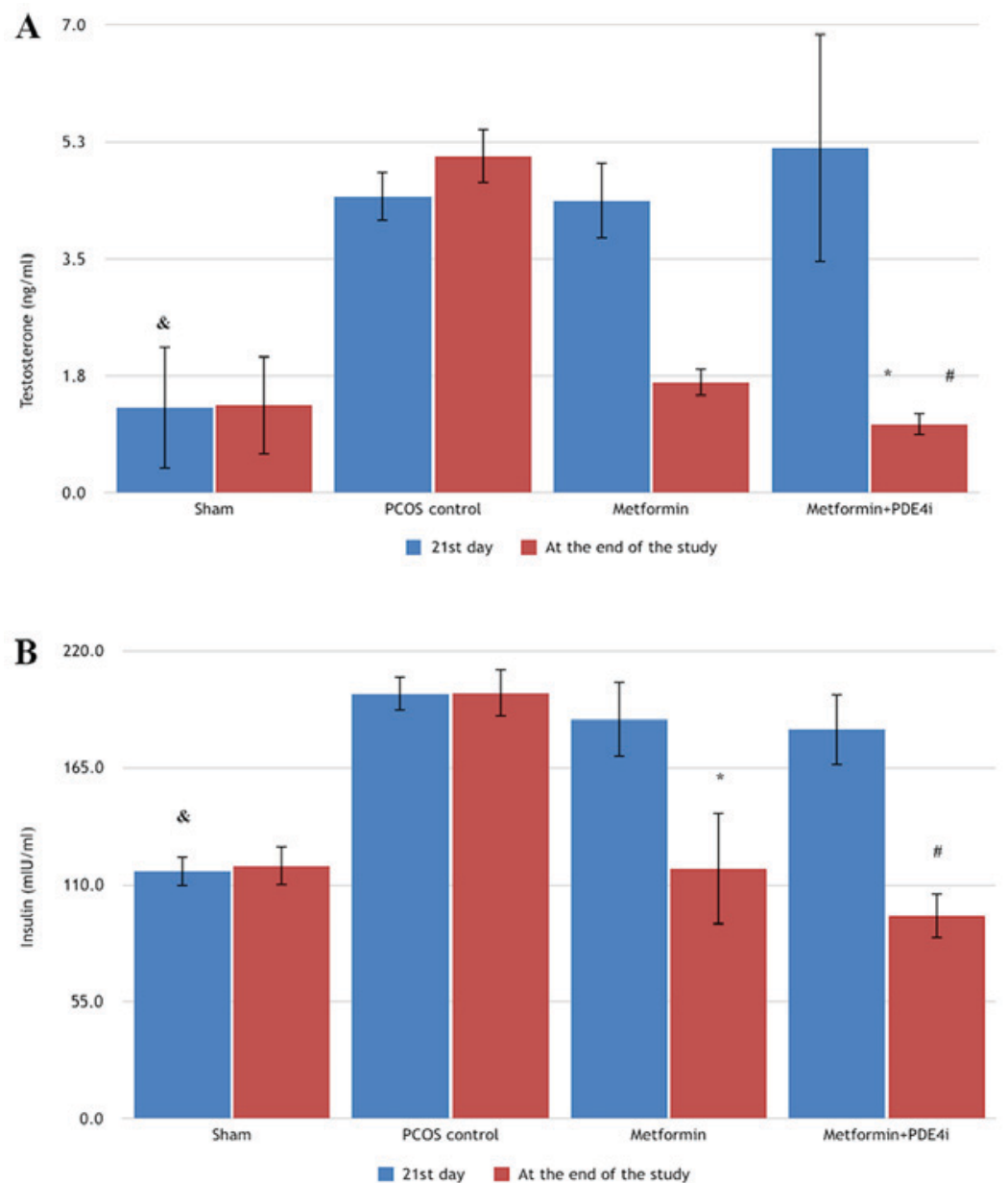

Figure 4. Testosterone and insulin levels following induction of PCOS and at the end of the study. (A) Testosterone levels. ${ }^{\circledR} \mathrm{P}<0.001$ compared with study groups (at the 21st day); ${ }^{\mathrm{P}}<0.001$ compared with the PCOS control group (at the end of the study); ${ }^{\text {"P}}=0.042$ compared with the Metformin group (at the end of the study); (B) Insulin levels. ${ }^{\text {\& }} \mathrm{P}<0.05$ compared with study groups (at the $21 \mathrm{st}$ day); ${ }^{\text {"P }}<0.001$ compared with the PCOS control group (at the end of the study); $\mathrm{P}=0.008$ compared with the PCOS control group (at the end of the study); PCOS, polycystic ovary syndrome; PDE4i, phosphodiesterase 4 inhibitor.
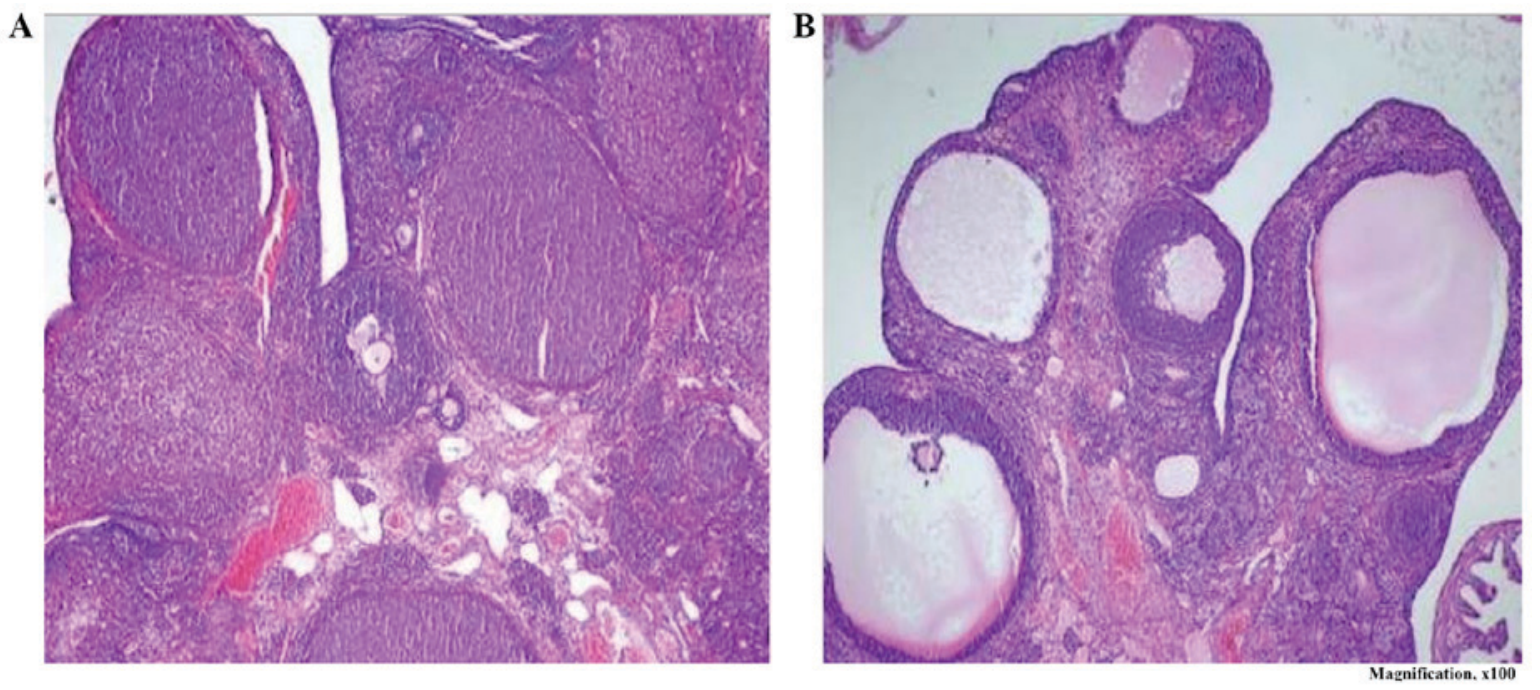

Figure 5. (A) Normal ovarian morphology in the sham, metformin and metformin + PDE4i groups; magnification, x100). (B) Numerous cystic follicles were identified in the PCOS control group (haematoxylin-eosin staining; magnification, x100). PCOS, polycystic ovary syndrome; PDE4i, phosphodiesterase 4 inhibitor.

is hypothesized to be responsible for reducing weight gain. According to earlier findings and those of the present study, the effects of PDE4i, including increasing the cAMP level, which leads to lipolysis, and increasing the GLP-1 level, which decreases the glucose level, may result in reduced weight gain, and PDE4i may be considered as a novel therapeutic option for 
Table IV. Testosterone levels at the end of the study.

\begin{tabular}{lccc}
\hline Groups & Median & IQR & Differences from 21 th day $^{\mathrm{a}}$ \\
\hline Sham $(\mathrm{n}=9)$ & 1.22 & 0.66 & $\mathrm{Z}=0.415 ; \mathrm{P}=0.678$ \\
PCOS control $(\mathrm{n}=9)$ & 4.88 & 0.65 & $\mathrm{Z}=2.547 ; \mathrm{P}=0.011$ \\
Merformin $(\mathrm{n}=8)$ & 1.67 & 0.36 & $\mathrm{Z}=2.521 ; \mathrm{P}=0.012$ \\
Metformin + PDE4i $(\mathrm{n}=10)$ & 1.05 & 0.22 & $\mathrm{Z}=2.803 ; \mathrm{P}=0.005$
\end{tabular}

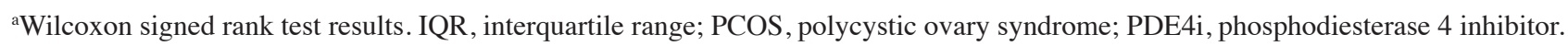

Table V. Insulin levels at the end of the study.

\begin{tabular}{|c|c|c|c|}
\hline Groups & Median & IQR & Differences from 21 th day \\
\hline Sham $(n=9)$ & 119.92 & 14.15 & $\mathrm{Z}=2.301 ; \mathrm{P}=0.021$ \\
\hline PCOS control $(n=9)$ & 194.52 & 18.91 & $\mathrm{Z}=0.059 ; \mathrm{P}=0.953$ \\
\hline Merformin $(n=8)$ & 106.64 & 53.97 & $\mathrm{Z}=2.521 ; \mathrm{P}=0.012$ \\
\hline Metformin + PDE4i $(n=10)$ & 97.12 & 17.52 & $\mathrm{Z}=2.803 ; \mathrm{P}=0.005$ \\
\hline
\end{tabular}

${ }^{\text {a} W i l c o x o n ~ s i g n e d ~ r a n k ~ t e s t ~ r e s u l t s . ~ P D E 4 i, ~ p h o s p h o d i e s t e r a s e ~} 4$ inhibitor; PCOS, polycystic ovary syndrome; IQR, interquartile range.
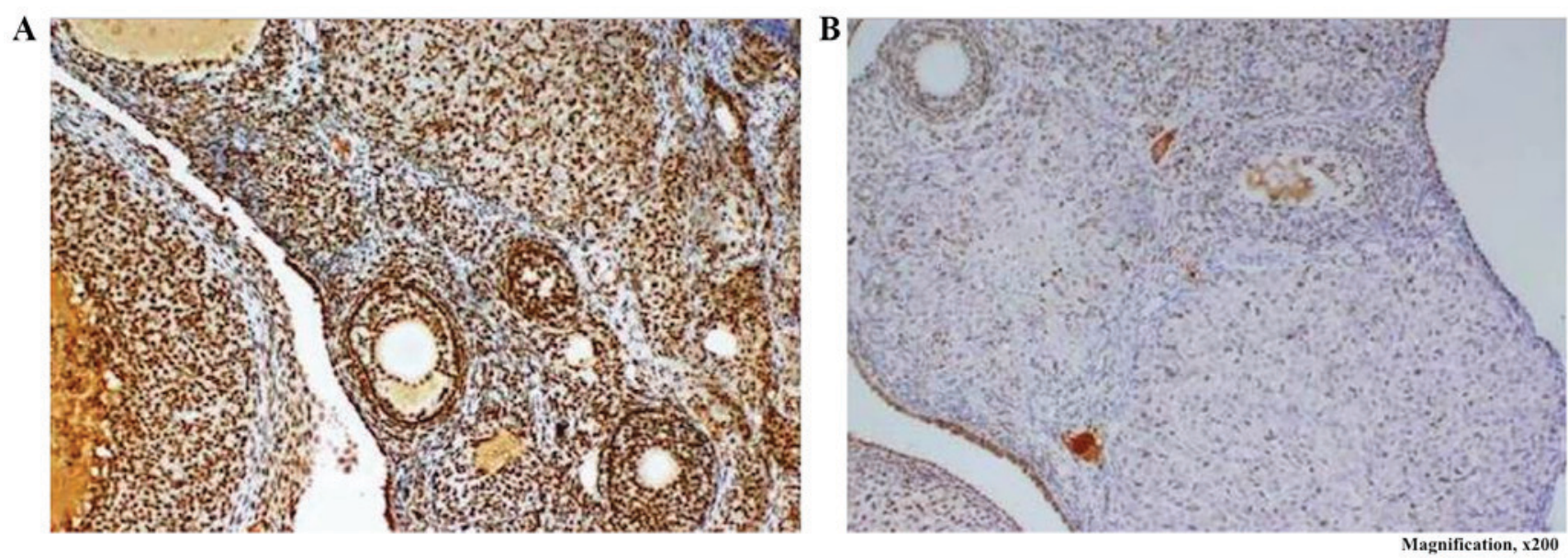

Figure 6. The expression of pCREB in ovarian tissue was examined by immunohistochemistry. (A) Strong expression was observed in the metformin + PDE4i group compared with (B) weak expression in the sham, PCOS control and metformin alone groups (pCREP; magnification, x200). PCOS, polycystic ovary syndrome; PDE4i, phosphodiesterase 4 inhibitor; pCREP, phosphorylated cyclic AMP response element-binding protein.

treating obesity and obesity-related diseases, such as PCOS; however, the weight loss potential of PDE4i in humans is not fully understood and must be elucidated via additional research.

PCOS is characterized by follicular developmental arrest without atresia during the small antral stage of folliculogenesis and, consequently, there is a lack of dominant follicle development and ovulation (34). The main cause of follicular arrest is disruption of the balance between androgens, gonadotropins and anti-Müllerian hormone (34). In cases with PCOS, the hormonal balance shifts to the androgen side and, as a result, causes anovulation, as ovarian theca cells produce excessive androgen with the additive effect of insulin-like growth factor- 1 and insulin on LH, increasing androgen production $(35,36)$. In addition, insulin can directly increase the activity of ovarian P450c17 and P450scc enzymes, which produce ovarian androgens (37). Similar to hyperinsulinemia, increased fat deposition and weight gain is positively correlated with hyperandrogenemia (38). These findings indicate that hyperinsulinemia and increased fat deposition and weight gain play an important role in hyperandrogenemia in PCOS cases; therefore, drugs with a beneficial effect on insulin and fat metabolism, such as metformin and PDE4i, may be useful for improving glucose and hormonal homeostasis in patients with PCOS.

The effects of gonadotropin, such as oocyte maturation and ovulation induction, are primarily associated with an increase in cAMP in ovarian follicles. The main sources of cAMP in ovarian follicles are cumulus cells, which primarily contain PDE4. The follicular cAMP level is primarily regulated by 
PDEs and increased by PDE4i $(39,40)$. According to these findings and those of the present study, PDE4i appears to regulate folliculogenesis and may prevent follicular arrest in polycystic ovaries, therefore exerting a positive effect on the menstrual cycle and hormonal balance; however, these effects were only observed in animal models, and the effects of PDE4i on human ovaries have not been fully elucidated; therefore, additional research is required to discern whether the effects on humans are similar.

There were certain limitations to the present study, including the lack of a PDE4i alone group. Although metformin has a modest effect in the management of PCOS and PCOS-related obesity, it is still the mainstay of the medical treatment. Therefore, as the present study only aimed to determine the effect of PDE4i added to metformin, its effect alone was not evaluated. In addition, the present study used an animal model and it is well-known that an experimental animal model may not completely simulate human disease; as such, the present findings must be confirmed in human research.

In conclusion, PDE4i added to metformin was superior to metformin alone in reducing weight gain, and also exerted a beneficial effect on insulin homeostasis. We hypothesized that the combination of these effects of PDE4i decreased the testosterone level in a rat model of PCOS. However, further investigation in humans is required to discern the value of PDE4i in combination with metformin for treating obesity-related conditions, such as PCOS.

\section{Acknowledgements}

Not applicable.

\section{Funding}

The present study was supported by the Başkent University Research Fund (project no. DA15/45).

\section{Availability of data and materials}

The datasets generated and analyzed during the present study are available from the corresponding author on reasonable request.

\section{Authors' contributions}

YAT, HBZ and GO contributed to the study concept and design. YAT, GO, MT and EC contributed to writing the manuscript. MT and NB acquired the data. GO, MT, NB, EEO and EC were involved in the analysis and interpretation of data. YAT, HBZ, GO, MT and EC drafted the manuscript. YAT, HBZ, $\mathrm{GO}, \mathrm{EEO}, \mathrm{NB}$ and MT were involved critically revising the manuscript for important intellectual content. HBZ performed the statistical analysis. All the authors have read and approved the final version of this manuscript.

\section{Ethics approval and consent to participate}

The study protocol was approved by the Başkent University Animal Care and Use Committee (project no. DA15/45), in accordance with Turkish legislation for animal experimentation.

\section{Patient consent for publication}

Not applicable.

\section{Competing interests}

The authors declare that they have no competing interests.

\section{References}

1. Stein IF and Leventhal ML Amenorrhea associated with bilateral polycystic ovaries. Am J Obstet Gynecol. 29: 181-191, 1935.

2. Carmina E, Napoli N, Longo RA, Rini GB and Lobo RA: Metabolic syndrome in polycystic ovary syndrome (PCOS): Lower prevalence in southern Italy than in the USA and the influence of criteria for the diagnosis of PCOS. Eur J Endocrinol 154: 141-145, 2006.

3. Broekmans FJ, Knauff EA, Valkenburg O, Laven JS, Eijkemans MJ and Fauser BC: PCOS according to the Rotterdam consensus criteria: Change in prevalence among WHO-II anovulation and association with metabolic factors. BJOG 113: 1210-1217, 2006.

4. Anagnostis P, Tarlatzis BC and Kauffman RP: Polycystic ovarian syndrome (PCOS): Long-term metabolic consequences. Metabolism 86: 33-43, 2018.

5. Li Q, Du J, Feng R, Xu Y, Wang H, Sang Q, Xing Q, Zhao X Jin L, He L and Wang L: A possible new mechanism in the pathophysiology of polycystic ovary syndrome (PCOS): The discovery that leukocyte telomere length is strongly associated with PCOS. J Clin Endocrinol Metab 99: E234-E240, 2014.

6. Alanbay I, Ercan CM, Sakinci M, Coksuer H, Ozturk M and Tapan S: A macrophage activation marker chitotriosidase in women with PCOS: Does low-grade chronic inflammation in PCOS relate to PCOS itself or obesity? Arch Gynecol Obstet 286: 1065-1071, 2012.

7. Ortega I, Villanueva JA, Wong DH, Cress AB, Sokalska A, Stanley SD and Duleba AJ: Resveratrol potentiates effects of simvastatin on inhibition of rat ovarian theca-interstitial cells steroidogenesis. J Ovarian Res 7: 21, 2014.

8. Noroozzadeh M, Behboudi-Gandevani S, Zadeh-Vakili A and Ramezani Tehrani F: Hormone-induced rat model of polycystic ovary syndrome: A systematic review. Life Sci 191: 259-272, 2017.

9. Bingham J, Sudarsanam S and Srinivasan S: Profiling human phosphodiesterase genes and splice isoforms. Biochem Biophys Res Commun 350: 25-32, 2006.

10. Vezzosi D and Bertherat J: Phosphodiesterases in endocrine physiology and disease. Eur J Endocrinol 165: 177-188, 2011.

11. Calverley PM, Rabe KF, Goehring UM, Kristiansen S, Fabbri LM and Martinez FJ; M2-124 and M2-125 study groups: Roflumilast in symptomatic chronic obstructive pulmonary disease: Two randomised clinical trials. Lancet 374: 685-694, 2009.

12. Tsai LC and Beavo JA: The roles of cyclic nucleotide phosphodiesterases (PDEs) in steroidogenesis. Curr Opin Pharmacol 11: 670-675, 2011.

13. Wouters EF, Bredenbröker D, Teichmann P, Brose M, Rabe KF, Fabbri LM and Göke B: Effect of the phosphodiesterase 4 inhibitor roflumilast on glucose metabolism in patients with treatment-naive, newly diagnosed type 2 diabetes mellitus. J Clin Endocrinol Metab 97: E1720-E1725, 2012.

14. Jensterle M, Kocjan T and Janez A: Phosphodiesterase 4 inhibition as a potential new therapeutic target in obese women with polycystic ovary syndrome. J Clin Endocrinol Metab 99: E1476-E1481, 2014.

15. Jin P and Xie Y: Treatment strategies for women with polycystic ovary syndrome. Gynecol Endocrinol 34: 272-277, 2018.

16. Jensterle M, Salamun V, Kocjan T, Vrtacnik Bokal E and Janez A: Short term monotherapy with GLP-1 receptor agonist liraglutide or PDE 4 inhibitor roflumilast is superior to metformin in weight loss in obese PCOS women: A pilot randomized study. J Ovarian Res 8: 32, 2015.

17. Anderson E, Lee MT and Lee GY: Cystogenesis of the ovarian antral follicle of the rat: Ultrastructural changes and hormonal profile following the administration of dehydroepiandrosterone. Anat Rec 234: 359-382, 1992. 
18. Mannerås L, Cajander S, Holmäng A, Seleskovic Z, Lystig T, Lönn $M$ and Stener-Victorin E: A new rat model exhibiting both ovarian and metabolic characteristics of polycystic ovary syndrome. Endocrinology 148: 3781-3791, 2007.

19. Wu XY, Li ZL, Wu CY, Liu YM, Lin H, Wang SH and Xiao WF. Endocrine traits of polycystic ovary syndrome in prenatally androgenized female Sprague-Dawley rats. Endocr J 57: 201-209, 2010.

20. Sander V, Luchetti CG, Solano ME, Elia E, Di Girolamo G, Gonzalez $\mathrm{C}$ and Motta AB: Role of the N, N'-dimethylbiguanide metformin in the treatment of female prepuberal BALB/c mice hyperandrogenized with dehydroepiandrosterone. Reproduction 131: 591-602, 2006.

21. Reagan-Shaw S, Nihal M and Ahmad N: Dose translation from animal to human studies revisited. FASEB J 22: 659-661, 2008.

22. Marcondes FK, Bianchi FJ and Tanno AP: Determination of the estrous cycle phases of rats: Some helpful considerations. Braz J Biol 62: 609-614, 2002.

23. Wang $\mathrm{H}$ and Edens NK: mRNA expression and antilipolytic role of phosphodiesterase 4 in rat adipocytes in vitro. J Lipid Res 48: 1099-1107, 2007.

24. Miao ZL, Guo L, Wang YX, Cui R, Yang N, Huang MQ, Qin WB, Chen J, Li HM, Wang ZN and Wei XC: The intervention effect of Rosiglitozone in ovarian fibrosis of PCOS rats. Biomed Environ Sci 25: 46-52, 2012.

25. Liu W, Liu W, Fu Y, Wang Y and Zhang Y: Bak Foong pills combined with metformin in the treatment of a polycystic ovarian syndrome rat model. Oncol Lett 10: 1819-1825, 2015.

26. Chang H, Jung WY, Kang Y, Lee H, Kim A and Kim BH Expression of ROR1, pAkt, and pCREB in gastric adenocarcinoma. Ann Diagn Pathol 19: 330-334, 2015.

27. Lafontan M and Berlan M: Fat cell adrenergic receptors and the control of white and brown fat cell function. J Lipid Res 34: 1057-1091, 1993

28. Doseyici S, Mehmetoglu I, Toker A, Yerlikaya FH and Erbay E: The effects of forskolin and rolipram on cAMP, cGMP and free fatty acid levels in diet induced obesity. Biotech Histochem 89: 388-392, 2014.

29. Snyder PB, Esselstyn JM, Loughney K, Wolda SL and Florio VA: The role of cyclic nucleotide phosphodiesterases in the regulation of adipocyte lipolysis. J Lipid Res 46: 494-503, 2005.

30. Nakamura J, Okamura N and Kawakami Y: Augmentation of lipolysis in adipocytes from fed rats, but not from starved rats, by inhibition of rolipram-sensitive phosphodiesterase 4. Arch Biochem Biophys 425: 106-114, 2004.
31. Zhang R, Maratos-Flier E and Flier JS: Reduced adiposity and high-fat diet-induced adipose inflammation in mice deficient for phosphodiesterase 4B. Endocrinology 150: 3076-3082, 2009.

32. Ong WK, Gribble FM, Reimann F, Lynch MJ, Houslay MD, Baillie GS, Furman BL and Pyne NJ: The role of the PDE4D cAMP phosphodiesterase in the regulation of glucagon-like peptide-1 release. Br J Pharmacol 157: 633-644, 2009.

33. Vollert S, Kaessner N, Heuser A, Hanauer G, Dieckmann A, Knaack D, Kley HP, Beume R and Weiss-Haljiti C: The glucose-lowering effects of the PDE4 inhibitors roflumilast and roflumilast-N-oxide in db/db mice. Diabetologia 55: 2779-2788, 2012.

34. Franks S, Stark J and Hardy K: Follicle dynamics and anovulation in polycystic ovary syndrome. Hum Reprod Update 14: 367-378, 2008

35. Willis D and Franks S: Insulin action in human granulosa cells from normal and polycystic ovaries is mediated by the insulin receptor and not the type-I insulin-like growth factor receptor. J Clin Endocrinol Metab 80: 3788-3790, 1995.

36. Lebbe M and Woodruff TK: Involvement of androgens in ovarian health and disease. Mol Hum Reprod 19: 828-837, 2013.

37. Adashi EY, Hsueh AJ and Yen SS: Insulin enhancement of luteinizing hormone and follicle-stimulating hormone release by cultured pituitary cells. Endocrinology 108: 1441-1449, 1981.

38. Dumesic DA, Akopians AL, Madrigal VK, Ramirez E, Margolis DJ, Sarma MK, Thomas AM, Grogan TR, Haykal R, Schooler TA, et al: Hyperandrogenism accompanies increased intra-abdominal fat storage in normal weight polycystic ovary syndrome women. J Clin Endocrinol Metab 101: 4178-4188, 2016.

39. Tsafriri A, Chun SY, Zhang R, Hsueh AJ and Conti M: Oocyte maturation involves compartmentalization and opposing changes of cAMP levels in follicular somatic and germ cells: Studies using selective phosphodiesterase inhibitors. Dev Biol 178: 393-402, 1996

40. Thomas RE, Armstrong DT and Gilchrist RB: Differential effects of specific phosphodiesterase isoenzyme inhibitors on bovine oocyte meiotic maturation. Dev Biol 244: 215-225, 2002 . International (CC BY-NC-ND 4.0) License. 